\title{
Co-designing engaging and accessible data visualisations: a case study of the IPCC reports
}

\author{
Angela Morelli ${ }^{1}$ (D) - Tom Gabriel Johansen ${ }^{1} \cdot$ Rosalind Pidcock $^{2,3,4} \cdot$ Jordan Harold $^{5,6}$ • \\ Anna Pirani $^{2} \cdot$ Melissa Gomis $^{2} \cdot$ Irene Lorenzoni $^{5,7}$ - Eamon Haughey ${ }^{8,9}$. \\ Kenny Coventry ${ }^{6}$
}

Received: 12 January 2021 / Accepted: 9 July 2021 / Published online: 26 October 2021

(C) The Author(s) 2021

\begin{abstract}
Creating scientifically rigorous and user-friendly data visualisations can play a critical role in making complex information more accessible to wider audiences and supporting informed decision-making. 'Co-design' encapsulates a way of approaching data visualisation that ensures a deep and shared understanding between those creating the visuals (e.g. information designers, content experts, cognitive scientists) and the audience/users. This essay describes co-designing data visualisations with the Intergovernmental Panel on Climate Change (IPCC). A multidisciplinary design team made up of information designers and cognitive and social scientists worked closely with IPCC authors and staff to develop data visualisations for the Summary for Policymakers (SPM) of the Special Report on Global Warming of $1.5^{\circ} \mathrm{C}$ and the Special Report on Climate Change and Land. In this essay, the authors consider the three crucial elements that underpin a successful co-design process - practical tools and a flexible method; cognitive science and psychology to better understand the needs of users; and the importance of trust and leadership. The authors reflect on the application of the co-design approach in an IPCC context, noting specific challenges and including recommendations for future IPCC reports. The mutual learning experience of the special reports indicates a shift towards a design culture within parts of the IPCC that recognises the value of telling a compelling visual story while retaining scientific integrity — an approach that has been retained for the Working Group I contribution to the Sixth Assessment Report.
\end{abstract}

Keywords Data visualisation $\cdot$ Co-design $\cdot$ IPCC $\cdot$ Information design $\cdot$ Cognitive science

This article is part of the topical collection "Climate Change Communication and the IPCC", edited by Saffron O'Neill and Roz Pidcock.

Angela Morelli

angela@infodesignlab.com

Extended author information available on the last page of the article 


\section{1) Data visualisation for decision-making and the IPCC context}

In our increasingly data-driven world, visualisations that illustrate complex scientific and technical data can help inform our everyday decisions. For example as patients, we need to understand whether or not to undergo medical treatment. As consumers, we can compare the energy performance of appliances to help us buy responsibly. Data visualisation harnesses the human visual system's capacity to be a powerful pattern detector. Data presented in visual forms can aid decision-making when it leverages our remarkable ability to process visual information.

But just as in verbal or written communication, there are more and less effective ways to communicate visually. For data visualisations to be effective decision-making tools that help us discern, distinguish, learn and understand, the design and production processes are not trivial. Increasingly, research insights from the fields of design, cognition, psychology and behavioural economics, as well as the increasing robustness of human-centred and participatory design methodologies, can guide us in designing data visualisations that enhance understanding, empower the final users and support informed decision-making at all levels.

The concept of 'co-design' is a major domain of design research and practice that has highlighted the importance of participation by all stakeholders in the design process (Aguirre 2020). This essay describes and reflects on the 'co-design' of data visualisations for reports produced by the UN body that assesses the science of climate change: the Intergovernmental Panel on Climate Change (IPCC). Drawing on tens of thousands of scientific publications, the IPCC undertakes regular assessments and produces special reports on the very latest advances in knowledge. For each assessment and report, the key findings are synthesised into a short Summary for Policymakers (SPM).

There are a number of distinct challenges to creating visuals for IPCC SPMs. Among other requirements, visuals need to be scientifically rigorous, explain scientific assessment and often integrate several lines of evidence while also being transparent and relevant for a growing mix of users (including policymakers who are the primary audience of the SPMs, international organisations, the media, communities and citizens around the world). Visual communication of complex climate data, especially the uncertainties associated with such data, is particularly challenging (Spiegelhalter et al. 2011; McMahon et al. 2015). In the past, the IPCC has been criticised for producing visuals that contain jargon and lack clarity, making them inaccessible and difficult to comprehend (Yeo 2013; Black 2015). On the other hand, IPCC visualisations need to avoid oversimplification of complex messages as 'simplistic' visuals can reduce readers' confidence in the scientific credibility of the information (McMahon et al. 2016). Thus, there is an important balance to be achieved between the scientific rigour of visuals and their visual appearance and appeal.

In the Sixth Assessment Cycle (AR6), the IPCC has dedicated considerable efforts to improving the accessibility of data visualisations within its reports, reflecting a growing recognition that these visuals - particularly in the SPMs - can be at least as important for communicating key findings as the text itself. Below we reflect on the process of co-design, drawing upon two occasions in which the relevant Working Groups of the IPCC chose to adopt a co-design approach to developing data visualisations: the Summaries for Policymakers (SPM) of both the Special Report on Global Warming of $1.5^{\circ} \mathrm{C}$ and the Special Report on Climate Change and Land. We outline and discuss the process by which a multidisciplinary design team made up of information designers and cognitive and social scientists worked closely with IPCC authors and staff to develop the data visualisations. Specifically, the authors 
discuss three elements of the co-design process that in their experience are key to success and their application in the specific IPCC context. These elements are (i) building the co-design process around the user and some of the practical tools used; (ii) using insights from cognitive science and psychology to guide and support the co-design process by helping to understand the needs of the users; and (iii) the importance of strong leadership, vision and mutual trust from the very beginning of the journey. The dynamics, challenges and opportunities are also considered. Of importance here is the acknowledgement that co-design requires flexibility. It does not follow a standard formula; rather, as the context, the challenges and the number of stakeholders involved vary within each process, co-design requires tailoring and revisiting according to specific circumstances.

\section{2) Element 1: Practical tools for co-designing data visualisations}

Defined as 'collective creativity' Sanders and Stappers 2008) and 'joint inquiry and imagination' (Steen 2013), co-design is a major domain of design research and practice that highlights the importance of participation in the design process (Aguirre 2020).

When the goal is to design scientifically rigorous data visualisations for decision-making, undertaking a 'co-design' approach opens up possibilities for ensuring the participation of information designers, users, content experts (hereafter referred to as 'authors', in the IPCC context), cognitive scientists and multiple stakeholders to achieve a deep and shared understanding of the nature of the data and the science that underpins it, as well as the assumptions, caveats, connections and communication challenges associated with it. Co-design is a methodology that has its roots in user experience (UX)/user interaction (UI), service design and system design. In the context of data visualisation, through participation (and a shared understanding), the co-design process aims to lay foundations for designing visuals that are accessible, clear and usable.

For meaningful co-design, it is critical to develop a method that can sustain a multi-stakeholder process and facilitate constructive discussions between parties that can be unfamiliar with visualisation concepts. This can be done using facilitation tools that support co-creation with the authors and by checking understanding of the co-produced material by the users. As such, the co-design process is organised to create maximum space for sharing, for conversations and feedback. Typically, a single figure goes through two or three iterations, with each iteration organised in three cycles: A, B and C (though the number is not set in stone). Each cycle, in turn, consists of a sequence of design meetings and design work (Fig. 1). User involvement is carefully planned within these cycles and the rhythm can be defined in line with content experts' availability and stakeholders' needs, ensuring maximum flexibility to meet the rigid timeline of the projects.

One of the most critical aspects for co-designing data visualisations and for ensuring constructive discussions around them is to establish the intent of the visual: that is, the goal that a visualisation aims to achieve is summarised in one short, plain language sentence. The intent might evolve along the co-design process through various stages of interaction, and once the intent is clear, it should be continually referred back to in order to measure the success of what the visualisation shows or agree on necessary changes after user testing.

In a multi-stakeholder context, a visualisation without a clear intent is at risk of becoming incrementally more complex since suggested changes that are unclear, counter-intuitive or motivated by personal opinions can result in a variety of conflicting or diverse elements represented in the figure. In the multistage co-design process described here (see Fig. 1), however, the number of iterations does not imply that the number of changes to the visual increases. Rather, 
Co-design of the SPM figur es - IPCC AR6 WG1

\section{RESEARCH and PRE-DESIGN PROCESS}

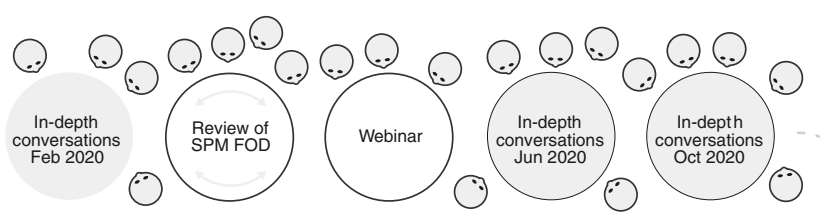

\section{DESIGN ITERATION 1}

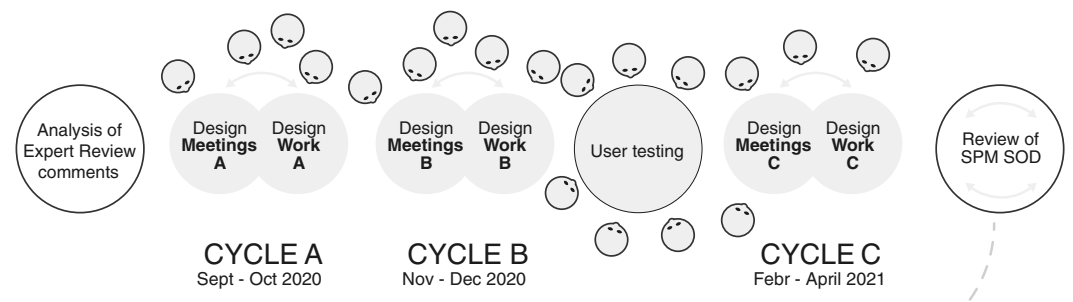

\section{DESIGN ITERATION 2}
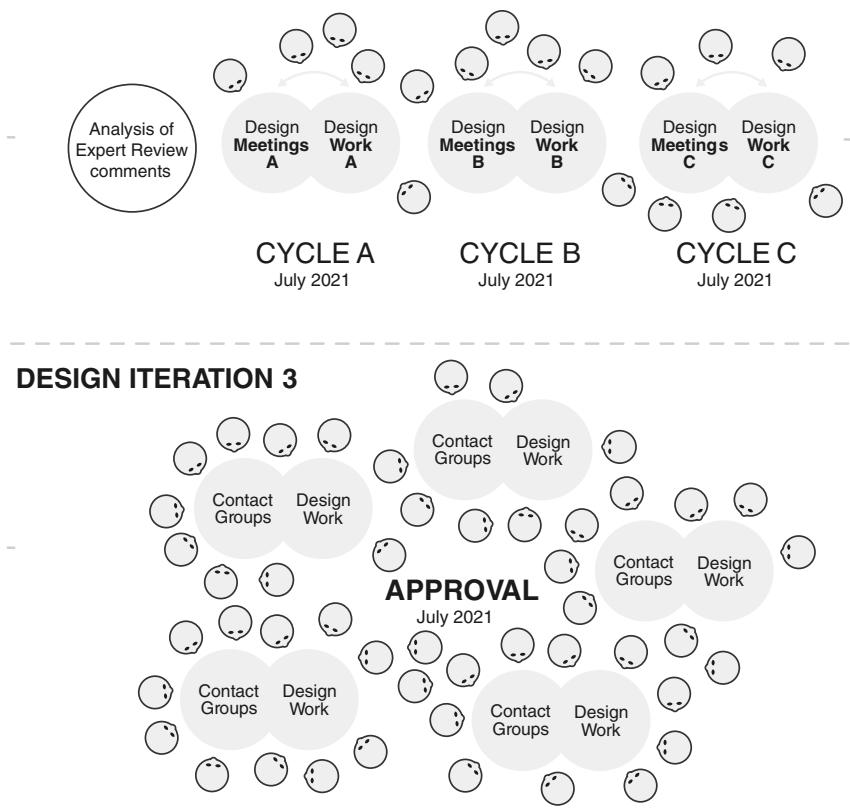

Fig. 1 The co-design process of the data visualisations for the IPCC Summary for Policymakers is a multistakeholder and iterative process, organised in cycles of design meetings, design work and user involvement. The process usually starts with user involvement during the review of the First Order Draft and ends with real-time design support during the final approval. Dates in the figure refer to the co-design process planned for the Working Group I contribution to the Sixth Assessment Report

each iteration is intended to bring the figure in closer alignment with the agreed intent and, as a result, to facilitate a continued appraisal of how the visual can best suit the user's needs. A 
commitment to revisiting the intent as the agreed benchmark against which any suggestions and discussions are measured requires discipline and compromise. But doing so not only ensures clarity and transparency around decision-making but engenders trust between members of the codesign team as parties work towards a common vision.

The visual narrative is what the visualisation shows in order to fulfil the intent. If the intent of a figure is clear, it is possible to set a clear information hierarchy, i.e. a prioritisation of information to be included in the visual. This, in turn, allows a visual narrative to be constructed around that information hierarchy. Space, colour, layout, typography and annotations are all used to guide the users through the experience of processing the information.

Intent and visual narrative undergo an intense and rigorously documented evolution based on the development of the report's narrative. Different figures might require a different number of iterations, with sometimes dozens of prototypes to arrive at a single final figure. As an example of some of the milestones that mark such a process, Figure $3 \mathrm{a}$ and $3 \mathrm{~b}$ in the Summary for Policymakers of the Special Report on Global Warming of $1.5^{\circ} \mathrm{C}$ illustrate the emission evolutions in $1.5^{\circ} \mathrm{C}$ pathways and the implications for overshoot. This figure was originally conceived as one figure and evolved through different layouts. Analysis of the review comments received on the single multi-panel figure in the Final Order Draft led to the design decision to separate the visual into two figures to improve accessibility and usability. The two figures had two different intents, one showing the characteristics of global emissions pathways (3A) and the other showing those of four illustrative pathways (3B). When the two figures entered the approval session, it became apparent that Figure 3B could be a powerful future policymaking tool and the table that complemented figure 3B was expanded with a number of policy-relevant global indicators.

Within the broad co-design method outlined above, user involvement is the baseline for a usercentred approach. In the design of data visualisations for the IPCC summaries for policymakers, five aspects of user involvement were key to shaping the outcomes. First, in-depth conversations with members of the target audience were planned at the very beginning of the co-design process to assess their needs and expectations. Secondly, follow-up conversations were planned, if needed, during the design iterations to provide a 'sense-check' on evolving draft visuals. Thirdly, user testing was planned via a survey with IPCC delegates, with a view to eliciting how individuals perceive and understand draft versions of the figures (see Element 2 section, below). Fourthly, IPCC reports go through a unique process involving several rounds of review and feedback by experts and governments worldwide. This meant several thousand review comments that the core design team needed to consider together with the authors to produce the figures to go in the next draft of the report. Each time the figures went through review or user testing, the design team gained greater insights into users' preferences and comprehension. Finally, the SPM is approved line by line by government delegations from all member states of the IPCC during the final approval session. The design team carried out real time design work, to ensure that the scientific information was as clear, complete, accurate and useful to policymakers as it could be. The final, approved data visualisations produced for the Special Report on Global Warming of $1.5^{\circ} \mathrm{C}$ and the Special Report on Climate Change and Land can be seen in Fig. 2.

\section{3) Element 2: Cognitive science and psychology to facilitate understanding of user comprehension}

A successful co-design approach requires a shared understanding of the 'intent' of the data visualisation, the format of information to be communicated and the audience for whom the 

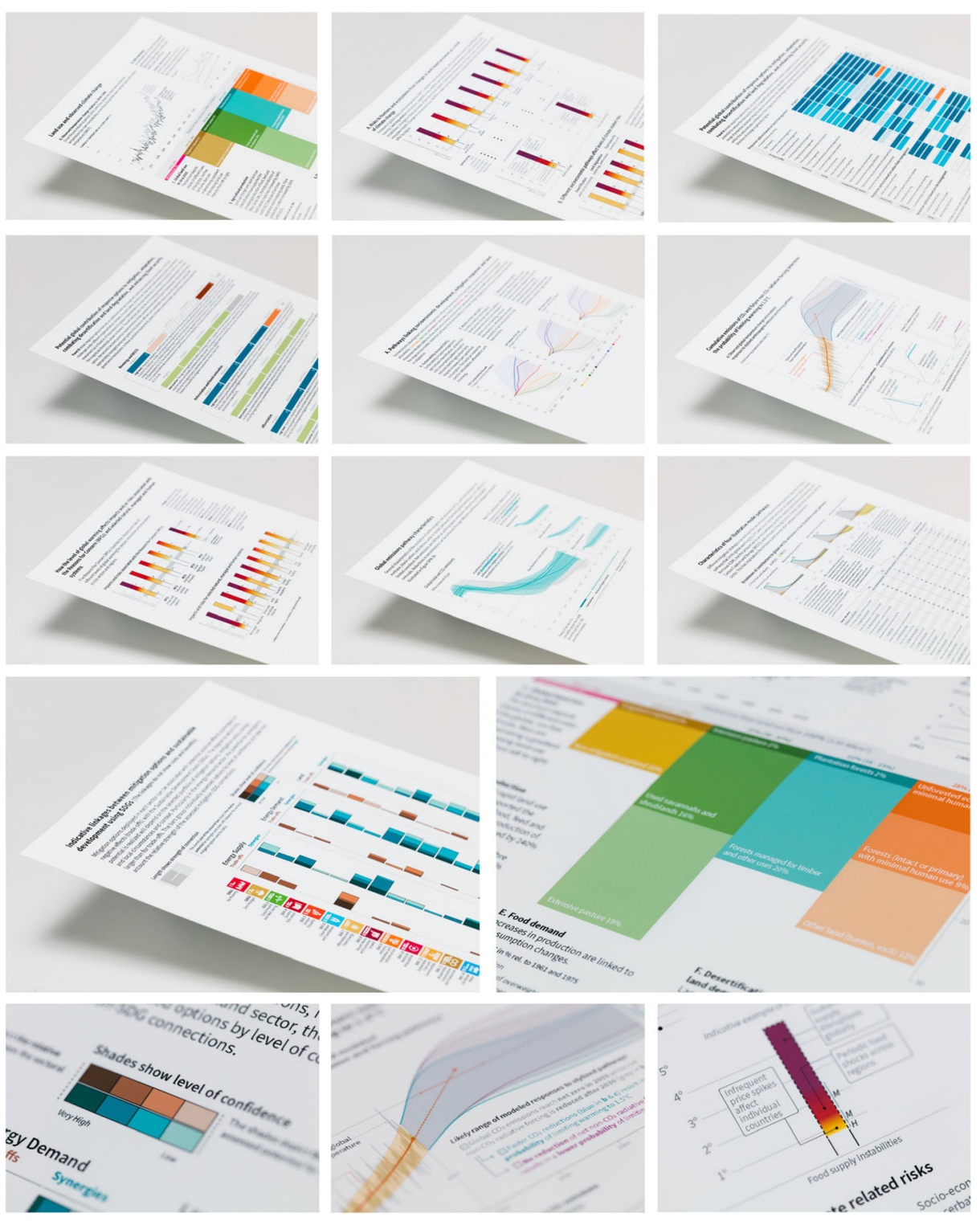

Fig. 2 The figures of the Summary for Policymakers of the Special Report on Global Warming of $1.5^{\circ} \mathrm{C}$ and the Special Report on Climate Change and Land. The figures were produced in various formats appropriate for online, download and outreach purposes

communication is intended. However, there are a multitude of ways in which information can be visually represented. Therefore, it is important that design choices consider 'how' potential audiences process visual information to help maximise ease of comprehension and usability. Embedding an evidence-based approach to the co-design process enables informed decisions to be taken on the visual representations of scientific information, such that data visualisations meet the requirements of those producing and using them. 
Insights from the cognitive and psychological sciences provide a rich evidence-base about how people process and create meaning from data visuals (Hegarty 2011; Harold et al. 2016; Padilla et al. 2018). At the level of the individual, comprehension involves the dynamic integration of incoming sensory information (driven by visual attention) with prior knowledge (formed from past experiences). Comprehension is facilitated when the incoming visual information matches an individual's expectations. Hence, data visualisations that employ familiar features whose meaning is well understood are more easily comprehended than those that use novel and unfamiliar features. Novel data visualisations with a high degree of complexity can be difficult to understand by non-specialists(Harold et al., 2020a, b). Crucially, design choices can influence comprehension and how information is integrated with prior beliefs (Bosetti et al. 2017).

Drawing on these cognitive insights, principles for good visual design, encapsulated by the 'MADE' principle (Table 1), were integrated into visual design guidance provided to IPCC authors (Gomis and Pidcock 2018). These principles provide a starting point for drafting accessible figures and include evidence-based design principles such as integrating and structuring text (Ginns 2006) and using colour sparingly to help direct readers' visual attention (Wolfe and Horowitz 2004). Reviewing draft figures in relation to these cognitive principles provided further support for the co-design process of both the IPCC special reports on Global Warming of $1.5^{\circ} \mathrm{C}$ and Climate Change and Land.

Embedding user evaluation is essential to understand whether information is understood as intended. We involved over 500 individuals in user evaluation, including IPCC delegates, policymakers, IPCC authors, researchers, representatives of NGOs and interested others. Evaluation can take a variety of forms according to the stage of the co-design process. For example, during IPCC plenary meetings, we invited delegates to take part in one-to-oneindepth conversations (semi-structured interviews) about their visual design preferences and the contexts in which they use IPCC figures. In doing so, we found that while text annotations were helpful to explain complex concepts to a reader, they also impaired the ease with which the visualisations could be re-used in presentations (Harold et al., 2020a, b). We also used surveys to evaluate how easy or difficult draft figures were to comprehend and to test alternative design ideas. Summaries of user evaluations were presented and discussed with figure author teams to feedback insights into the co-design process.

In an ideal world, quantitative testing of text and figures with target users should take place iteratively, throughout the production process, with stratified sampling methods and sample sizes powerful enough to ensure robust statistical evaluation. Qualitative insights from users, for instance via interviews, are also valuable to understand how texts and figures are perceived and used; these views help to inform iterative stages of co-design processes. It is also important that the testing process be as inclusive as possible to encapsulate the views and voices of the diverse users of IPCC reports. In practice, there are often time constraints that can limit testing time and the number of iterative cycles available, requiring prioritisation of what to test. In

Table 1 The MADE principle, to support evidence-based communications design (Harold et al. 2017)

\begin{tabular}{ll}
\hline Principle & Associated prompt \\
\hline Message & Does the visual communicate a clear message? \\
Audience & Is the visual appropriate for the intended audience(s)? \\
Design & Does the visual use evidence-based design principles? \\
Evaluation & Has the visual been tested with the audience(s)? \\
\hline
\end{tabular}


addition, drafts of IPCC reports are confidential during the drafting process, limiting, to some extent, the audiences that draft visuals can be tested with.

Acknowledging the relevance and importance of the co-design process has enabled the introduction of iterative user evaluation of visuals during the production of the most recent IPCC special reports. The outputs of this approach have facilitated a better understanding of the audiences of IPCC reports, the identification of comprehension and usability issues and evaluation of solutions to overcome such challenges, resulting in improved figures that better convey the intent of the visual and better meet the needs of the audience.

\section{4) Element 3: The importance of strong leadership, vision and mutual trust}

A participatory and multidisciplinary design approach always requires strong leadership and steer in what is a scientifically and technically demanding process. The strict timeline of the IPCC assessment process with several immovable deadlines means that the time available for producing a coherent set of SPM visuals is short, yet a carefully planned and iterative process needs to be implemented. The design process must also respond and adapt to the evolving nature of the underlying scientific assessment, as it undergoes multiple formal review stages and assimilates the latest scientific publications.

All author teams remained committed and engaged in the co-design process through to approval. It is, however, important to recognise that the co-design process is demanding of the authors as it requires them to converge on a clear intent for the data visualisations which is fully reflective of the outcomes of up to 2 years' worth of work on the assessment. They are challenged by this integrative process of identifying the key policy relevant information to prioritise for the SPM and to visually communicate key messages with scientific rigour to policymakers. In addition to becoming familiar with the scientific information, the design team must undertake multiple design steps with the authors that include detailed consultations on the assessment outcomes and the underlying data to fully understand the process by which it has been collected and processed. In addition, the IPCC process requires a transparent assessment, and authors must retain ownership of the data visualisations.

The TSU that implements the assessment process needs to ensure that review comments submitted by governments and experts are all discussed, taken into account where relevant, and fully responded to by authors. The unique approval process of IPCC reports, in which the SPM visuals undergo scrutiny and real-time modification, requires intensive TSU coordination to produce a final iteration that is realistic in scope for authors and the design team to complete under extremely demanding circumstances. In guiding the overall process, the TSU works to ensure that all perspectives are heard, thoroughly documenting discussions, and taking key decisions, in consultation with IPCC leadership, on the direction and development of the SPM.

Especially in contexts where there are a large number of stakeholders involved, leadership is important to ensure that there is a strong foundation of mutual trust between design team and content experts, in order to design together with the final users. In such a complex context, leadership ensures that flexibility, openness to new ideas and adaptability to sudden changing demands are accepted with confidence. Balancing these elements alongside competing pressures was, at times, extremely challenging and required patience on all sides, as well as strong facilitation. But the value of investing the time and commitment has not only resulted in figures that are more accessible and usable for the intended audience, but also an improved 
appreciation for user-led design among IPCC authors and staff. The learning journey may have a positive impact in the wider scientific community, empowering scientists or scientific organisations with a new mindset and tools when it comes to the presentation of complex data.

\section{5) Reflections, next steps and recommendations}

Undertaking a 'co-design' approach in data visualisation can ensure the participation of users, content experts, information designers, cognitive scientists and multiple stakeholders to design visualisations that enhance understanding, empower the final users and support informed decision-making.

While the IPCC experience is about designing with scientists for policymakers, co-design has the potential to impact any kind of decision-making, ranging from local to international scales and in any discipline. From sustainability to social justice, from education to health, codesign can enable people to see patterns, think systematically about the challenges we face as a society, inspire leadership, find solutions and, ultimately, act responsibly on them.

A co-design process is not a plug-and-play formula, but rather a flexible blueprint that has to be tailored according to the project's goals and ambition, to the diversity of the extended design team, to the number of stakeholders involved, time and resource availability and to the communication challenges.

The co-design process is a learning journey for the organisations and stakeholders involved that contributes not only to design solutions that empower the final users but also to a change in culture by increasing the collaboration across silos within an organisation and across different disciplines. As such, a co-design and evidence-based approach has been adopted for the Working Group I contribution to the Sixth Assessment Report.

Iterative processes of co-design can be complex and resource intensive; but in our experience, challenges can often be overcome through experimentation, openness and creativity, as well as honest reflection to help identify lessons learnt for the future. In summary, drawing on our experiences of co-design, key recommendations for creating engaging and accessible data visualisations are:

- Embed co-design methods early on in the process to bring together content experts and content users and to facilitate constructive dialogues.

- Establish the intent of the visual (the goal that the visual aims to achieve) as a benchmark against which decisions are made.

- Develop a visual narrative - prioritise what information needs to be shown in the visual to achieve the intent.

- Iteratively evaluate and user-test drafts of the visual and loop insights back into the codesign process.

- Provide strong leadership to develop a shared vision and to establish mutual trust between all co-design participants.

Acknowledgements The authors would like to thank all IPCC authors, TSU staff and Bureau members who participated in the co-design of data visualisations for the Special Reports on $1.5^{\circ} \mathrm{C}$ and Climate Change and Land.

Code availability Not applicable. 
Funding The work described here on the IPCC Special Report on Global Warming of $1.5{ }^{\circ} \mathrm{C}$ and the IPCC Special Report on Climate Change and Land was funded by the Technical Support Units of IPCC WGI and WGIII, respectively.

Data availability Not applicable.

\section{Declarations}

Ethics approval Ethical approval for user evaluation activities was sought and received from the University of East Anglia Ethics committee in accordance with the Helsinki declaration and associated international standards.

\section{Consent to participate Not applicable}

\section{Consent for publication Not applicable}

Open Access This article is licensed under a Creative Commons Attribution 4.0 International License, which permits use, sharing, adaptation, distribution and reproduction in any medium or format, as long as you give appropriate credit to the original author(s) and the source, provide a link to the Creative Commons licence, and indicate if changes were made. The images or other third party material in this article are included in the article's Creative Commons licence, unless indicated otherwise in a credit line to the material. If material is not included in the article's Creative Commons licence and your intended use is not permitted by statutory regulation or exceeds the permitted use, you will need to obtain permission directly from the copyright holder. To view a copy of this licence, visit http://creativecommons.org/licenses/by/4.0/.

\section{References}

Aguirre UM (2020) Transforming public organizations into co-designing cultures: a study of capacity-building programs as learning ecosystems. The Oslo School of Architecture and Design 10:31 https://aho.brage.unit. no/aho-xmlui/handle/11250/2654789. Accessed 23 Sept 2020

Black R (2015) No more summaries for wonks. Nat Clim Chang 5:282-284

Bosetti V, Weber E, Berger L, Budescu DV, Liu N, Tavoni M (2017) COP21 climate negotiators' responses to climate model forecasts. Nat Clim Chang 7(3):185-191

Ginns P (2006) Integrating information: a meta-analysis of the spatial contiguity and temporal contiguity effects. Learn Instr 16(6):511-525

Gomis MI, Pidcock R (2018) IPCC visual style guide for authors. Published by IPCC WGI Technical Support Unit. https://www.ipcc.ch/site/assets/uploads/2019/04/IPCC-visual-style-guide.pdf. Accessed in December 2020

Harold J, Lorenzoni I, Shipley TF, Coventry KR (2016) Cognitive and psychological science insights to improve climate change data visualization. Nat Clim Chang 6(12):1080-1089

Harold J, Lorenzoni I, Coventry KR, Minns A (2017) Enhancing the accessibility of climate change data visuals: recommendations to the IPCC and guidance for researchers. Report published by the Tyndall Centre for Climate Change Research, Norwich, UK. https://www.tyndall.ac.uk/datavisuals. Accessed in December 2020

Harold J, Lorenzoni I, Coventry KR, Johansen TG, Morelli A, Gomis M, Pirani A (2020a) IPCC figures research at the fifty-second session of the IPCC (IPCC-52). Report published by the Tyndall Centre for Climate Change Research, Norwich, UK for IPCC WG1

Harold J, Lorenzoni I, Shipley TF, Coventry KR (2020b) Communication of IPCC visuals: IPCC authors' views and assessments of visual complexity. Clim Chang 158(2):255-270

Hegarty M (2011) The cognitive science of visual-spatial displays: implications for design. Top Cogn Sci 3(3):446-474

McMahon R, Stauffacher M, Knutti R (2015) The unseen uncertainties in climate change: reviewing comprehension of an IPCC scenario graph. Clim Chang 133:141-154

McMahon R, Stauffacher M, Knutti R (2016) The scientific veneer of IPCC visuals. Clim Chang 138:369-381

Padilla LM, Creem-Regehr SH, Hegarty M, Stefanucci JK (2018) Decision making with visualizations: a cognitive framework across disciplines. Cognitive Research: Principles and Implications 3(1):29 
Sanders E, Stappers PJ (2008)Co-creation and the new landscapes of design. CoDesign 4(1):5-18

Spiegelhalter D, Pearson M, Short I (2011) Visualizing uncertainty about the future. Science 333:1393-1400

Steen M (2013)Co-design as a process of joint inquiry and imagination. Des Issues 29(2):16-28

Wolfe JM, Horowitz TS (2004) What attributes guide the deployment of visual attention and how do they do it? Nat Rev Neurosci 5(6):495-501

Yeo S (2013) 'Science is not finished until it's communicated' - UK chief scientist. Climate Home News. October 3. Available at https://www.climatechangenews.com/2013/10/03/science-is-not-finished-until-itscommunicated-uk-chief-scientist/. Accessed 5 Oct 2020

Publisher's note Springer Nature remains neutral with regard to jurisdictional claims in published maps and institutional affiliations.

\section{Affiliations}

\section{Angela Morelli ${ }^{1} \cdot$ Tom Gabriel Johansen $^{1} \cdot$ Rosalind Pidcock $^{2,3,4} \cdot$ Jordan Harold $^{5,6}$. Anna Pirani $^{2} \cdot$ Melissa Gomis $^{2} \cdot$ Irene Lorenzoni $^{5,7} \cdot$ Eamon Haughey $^{8,9} \cdot$ Kenny Coventry $^{6}$}

1 InfoDesignLab, Oslo, Norway

2 IPCC Working Group I Technical Support Unit, Université Paris Saclay, Gif-sur-Yvette, France

3 Climate Outreach, Oxford, UK

4 Chichester, UK

5 Tyndall Center for Climate Change Research, University of East Anglia, Norwich Research Park, Norwich, UK

6 School of Psychology, University of East Anglia, Norwich Research Park, Norwich, UK

7 School of Environmental Sciences, University of East Anglia, Norwich Research Park, Norwich, UK

8 IPCC Working Group III Technical Support Unit, Imperial College London, London, UK

9 Botany Department, Trinity College Dublin, Dublin, Ireland 